\title{
Mitochondria and neurodegenerative diseases: Special issue of BMB Reports in 2020
}

Mitochondria is essential to generate metabolic energy in eukaryotic cells as well as to regulate calcium buffering, cell signaling, the production of reactive oxygen species (ROS), and apoptosis. They mainly produce most of the cellular energy derived from the breakdown of carbohydrates and fatty acids, which is consequently converted to ATP via oxidative phosphorylation. Mitochondria are also distinctive among the cytoplasmic organelles in that they contain their own DNA, which encodes limited number of mitochondrial proteins, tRNAs, and rRNAs.

Evidence has accumulated from many reports, indicating that mitochondrial abnormalities are involved in age-related neurodegenerative diseases (NDDs). Causal factors for most age-related neurodegenerative diseases, including Alzheimer's disease (AD), Parkinson's disease (PD), and amyotrophic lateral sclerosis are largely unknown. Although genetic defects are reported to cause a small number of NDDs, cellular, molecular, and pathological mechanisms of disease progression and selective neuronal cell death are not understood fully in these diseases. Especially, age-dependent and mitochondriagenerated ROS has been identified as an important factor that is responsible for disease progression and cell death, particularly in late-onset diseases. Based on the current hypothesis supported by many recent findings, this issue discusses the roles of mitochondria in the progression of age-related neurodegenerative diseases, the connection between mitochondrial abnormalities and NDD, and the drug development targeted to mitochondria in NDDs.

Dr. Kyung-Tai Min of Ulsan National Institute of Science and Technology firstly reviewed the most advanced techniques for investigating mitochondrial gene expression. In their review, a wide range of techniques available for investigating the mitochondrial genome, mitochondrial transcription, and mitochondrial translation are discussed that provide a useful guide to understanding mitochondrial gene expression.

A chronic inflammation is involved in $A D$ pathological processes through the innate immune cells including microglia, astrocytes, and other peripheral blood cells. Dr. Inhee Mook-Jung from Seoul National University College of Medicine reviewed the currently available candidates of peripheral biomarkers related with abnormal inflammation in $A D$ and their putative working mechanisms. In addition, they summarized

https://doi.org/10.5483/BMBRep.2020.53.1.310

Received 16 December 2019 the current state of the inflammatory biomarker researches in both clinical practice and molecular parameters.

Dr. Seong-Woon Yu of Daegu Gyeongbuk Institute of Science and Technology reviewed the novel role of translocator protein (TSPO) in the neuroinflammation. TSPO is a transmembrane protein located on the outer mitochondria membrane and mainly expressed in glial cells in the brain. In the review, they summarized the recent controversial findings and the discrepancy between pharmacological and genetic studies of TSPO.

Drs. Hee Kyung Jin and Jae-sung Bae of Kyungpook National University discussed the functional role of sphingolipids in neuroinflammation and its value as the potential target for diagnosis and therapy. Sphingolipids are ubiquitous building blocks of eukaryotic cell membranes that function as signaling molecules for regulating a diverse range of cellular processes. Recently, several studies have highlighted the pivotal role of sphingolipids in neuroinflammatory regulation. In addition, accumulating evidence points to sphingolipid engagement in neuroinflammatory disorders, including $A D$ and PD. This review would help the readers to understand the potential of sphingolipids as a new therapeutic and diagnostic target for neuroinflammatory diseases.

Recent studies have reported that mitochondrial defects are found in AD and worsen AD symptoms. Dr. Yong-Keun Jung of Seoul National University next discussed the new perspectives on mitochondrial dysfunction and inflammation in AD. As described previously, mitochondrial dysfunction-driven generation of ROS and their contribution to neuronal damage has been widely studied in AD pathogenesis. Moreover, ROS produced upon failure of mitochondrial quality control may be linked to the inflammatory response and influence the progression of $\mathrm{AD}$. Based on this background, the authors especially focused on inflammatory pathways that are associated with and initiated through defective mitochondria. In the present they summarized recent progress on the role of mitochondria-mediated inflammation in AD. Furthermore, it was discussed how reducing mitochondrial dysfunctionmediated inflammation could affect AD.

Various therapeutic agents targeting the mitochondrial pathways associated with $\mathrm{AD}$ have been developed over the past decade. Dr. Ae Nim Pae of Korea Institute of Science and Technology discussed the link between mitochondrial dysfunction and $\mathrm{AD}$ and its prospects for therapeutic intervention. In the review, they provided a detailed description of AD-related mitochondrial pathways and their small-molecule

ISSN: 1976-670X (electronic edition)

Copyright (C) 2020 by the The Korean Society for Biochemistry and Molecular Biology

c) This is an open-access article distributed under the terms of the Creative Commons Attribution Non-Commercial License (http://creativecommons.org/licenses/by-nc/4.0) which permits unrestricted non-commercial use, distribution, and reproduction in any medium, provided the original work is properly cited. 
modulators that have therapeutic potential. They focused on recently reported examples, and also overviewed the current challenges and future perspectives of ongoing research.

Lastly, Mr. Woo Hyun Shin and Joon Hyung Park in my laboratory and I have reviewed the regulatory function of p62 between ubiquitin proteasome system and autophagy as well as its role in the mitophagy and PD. The ubiquitin-proteasome system (UPS) and autophagy are two major degradative pathways of proteins in eukaryotic cells. Although these autophagy and UPS pathways have not been considered to be directly related, many recent studies proposed their close link and dynamic interconversion. In this review, we particularly focused on and summarized several regulatory molecules, including p62, which function in both UPS and autophagy and their crosstalk. In addition, the functional role of p62 in the mitophagy and its link to the pathogenesis of PD was briefly summarized.

In conclusion, tremendous progress has been made to reveal the cellular functions of mitochondria, but it is still in its infancy to discover their detailed roles during the progression of NDDs. I wish that the reviews in this issue are useful to expand much dynamic role of mitochondria and the understanding of its contribution to NDDs.

\section{Kwang Chul Chung, Ph.D.}

Department of Systems Biology, College of Life Science and Biotechnology, Yonsei University, Seoul 03722, Korea

E-mail: kchung@yonsei.ac.kr 